\title{
Drug-induced leukocytoclastic vasculitis: tigecycline a rare cause
}

\author{
Kalpana Bhairavarasu MD, Satish Mocherla MD, Jaya Amaram MD, \\ Ena Sharma MD, Philip A Conlin MD, Imran Umer MD
}

\begin{abstract}
Drug-induced leukocytoclastic vasculitis is an inflammation of blood vessels triggered by various drugs. It presents with a localized skin rash but may involve the internal organ systems, including the gastrointestinal tract, kidneys, lungs, central nervous system, and joints. The clinical recognition of drug-induced vasculitis is very important because continued use of the culprit drug can be organ or life threatening. The prognosis is excellent if the disease is limited to the skin and diagnosed promptly. The use of tigecycline has recently increased due to resistance patterns of bacteria, and it is important to recognize this potential adverse effect of this drug and to diagnose and treat the patient early to achieve a favorable outcome. To best of our knowledge, we report the first case of tigecycline-induced leukocytoclastic vasculitis.
\end{abstract}

Key words: Drug-induced vasculitis, leukocytoclastic vasculitis, tigecycline-induced vasculitis, tigecycline related leukocytoclastic vasculitis

\section{INTRODUCTION}

Vasculitis is defined as an inflammatory disorder of blood vessels characterized by pathological change in the structure and function with resultant narrowing, weakening, and scarring of the blood vessel wall. ${ }^{1}$ Drug-induced vasculitis is usually a small vessel vasculitis with leukocytoclastic vasculitis (LCV) histology. Antibiotics that commonly cause LCV include penicillins, aminopenicillins, sulfonamides, and quinolones. ${ }^{1}$ To best of our knowledge, this is the first reported association of LCV with tigecycline.

Corresponding author: Imran Umer MD

Contact Information: Imran.umer@ttuhsc.edu DOI: 10.12746/swrccc2015.0309.123

\section{Case Presenttion}

A 21-year-old man with a history of chronic left leg ulcer secondary to a motor vehicle accident with multiple skin grafts was admitted with painful swelling in both inguinal regions for three weeks. This was associated with fever and chills. There was no history of recent trauma, insect bites, or skin rash. His review of systems was unremarkable. Examination revealed a temperature $101{ }^{\circ} \mathrm{F}$, heart rate 86 beats/minute, and respiratory rate $16 /$ minute, and blood pressure $116 / 78 \mathrm{mmHg}$. He had bilateral enlarged tender inguinal lymph nodes soft to firm in consistency. His left leg had a $4 \mathrm{~cm}$ chronic ulcer with regular margins and no drainage. The rest of the physical examination was within normal limits. The patient was started on intravenous (IV) piperacillin/tazobactam for suspected sepsis. Later the piperacillin/tazobactam was switched to IV tigecycline on day five. An Infectious Disease consult broadened the empiric antibiotics 
coverage to include vancomycin and meropenem; tigecycline was continued. A fine needle aspiration of the inguinal lymph nodes was negative for malignancy, and an excisional biopsy of the inguinal lymph nodes showed chronic inflammation with no granulomas. On hospital day seven the patient started developing a new skin rash on both lower extremities which progressively worsened over the next 48 hours (Figure 1). A rheumatology consultation suspected vasculitis secondary to tigecycline after a thorough chart review. The patient was then started on systemic corticosteroids and colchicine, and on day nine tigecycline was discontinued. Extensive laboratories studies, including an immunologic workup with antinuclear antibodies (ANA), cytoplasmic antineutrophil cytoplasmic antibodies (C-ANCA), proteinase 3 (PR3) antibodies, myeloperoxidase (MPO) antibodies, antidouble-stranded DNA antibodies (Anti-dsDNA), were negative. A skin biopsy of the rash reported perivascular inflammatory cells with necrosis of vessel walls and deposition of fibrinoid material consistent with "leukocytoclastic vasculitis" (Figure 2). The patient's blood, sputum, and urine cultures were negative. On hospital day 11, his skin rash started resolving. Later the patient developed acute pericarditis with massive effusion on day 12. A cardiothoracic surgeon created a surgical window; the pericardial biopsy showed nonspecific inflammatory cells. The patient had a complicated hospital course, and on day 30 he developed acute pancreatitis, a known adverse effect of tigecycline. Subsequently he developed multiorgan failure and did not survive.

\section{Discussion}

LCV, also known as hypersensitivity vasculitis, is a relatively common condition. It can occur as a primary disorder or in association with drugs, infections, collagen-vascular diseases, hematologic disorders, and malignancy. ${ }^{2}$ The American College of Rheumatology proposed criteria to define leukocytoclastic vasculitis which include: patient age greater than 16 , use of a possible drug in temporal relation to symptoms, palpable purpura, maculopapular skin lesions, biopsy of a skin lesion showing neutrophils

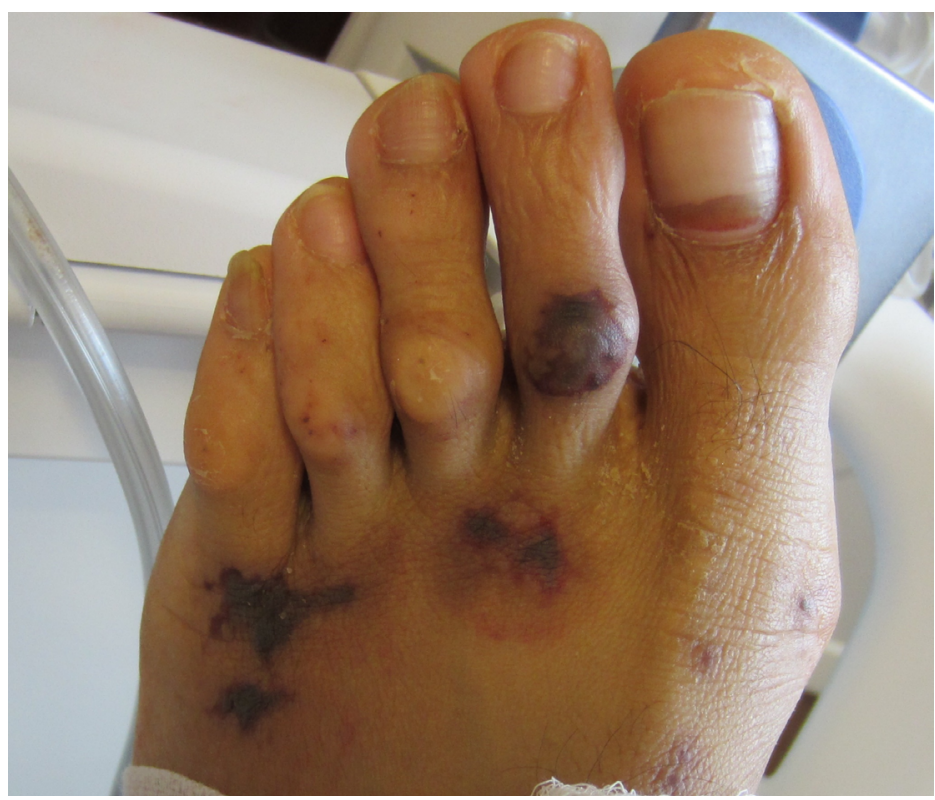

Figure 1 is a photograph of the macular purpuric rash on the left foot.

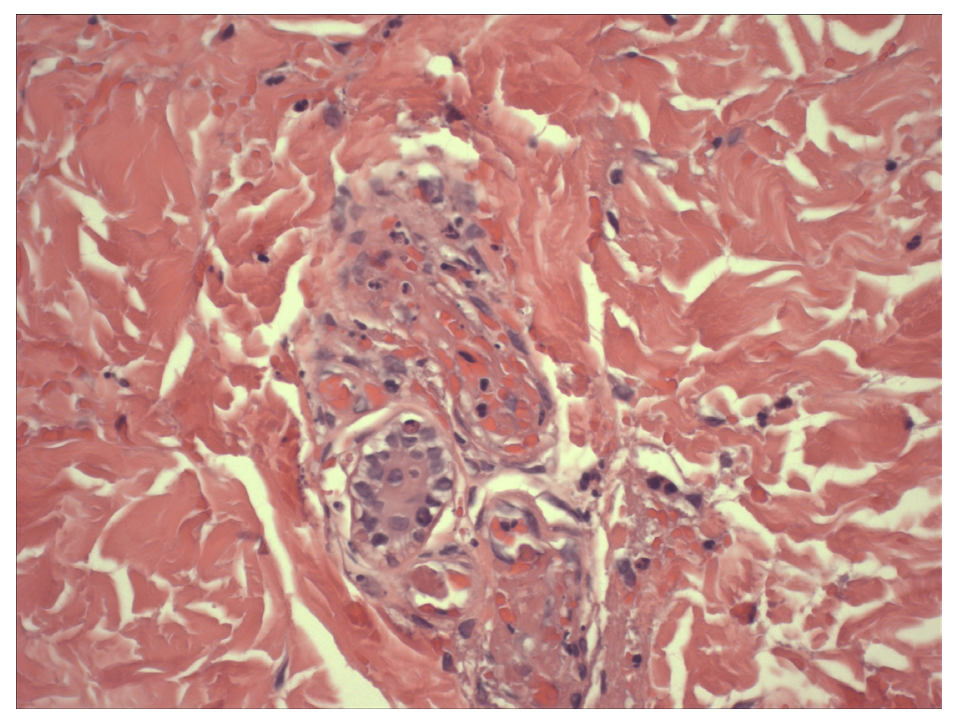

Figure 2 shows neutrophils infiltration around blood vessels with fibrinoid necrosis.

around arterioles or venules. At least three of five criteria must be present to diagnose LCV. ${ }^{3}$ Drug-induced LCV accounts about $10 \%$ of all vasculitis cases, and penicillins, sulfonamide, quinolones, allopurinol, propylthiouracil, valproic acid, phenytoin, anti-TNF alpha agents, and hydralazine are well known causes 
of LCV. ${ }^{4,5}$ The exact pathogenesis of LCV is unclear. Drugs may act as haptens to stimulate immune responses and a cascade of inflammation involving small vessels with deposition of immune complex in the walls of arterioles and postcapillary venules. In most cases it is type III immune complex-mediated reaction. ${ }^{6,7}$

The clinical manifestations of LCV vary from asymptomatic skin rashes to life threatening systemic involvement. ${ }^{8}$ It usually presents with a palpable purpuric or petechial skin rash involving the legs and can be associated with skin ulceration or edema. LCV can also involve internal organs, including the gastrointestinal tract, kidneys, lungs, central nervous system, and joints, and can be fatal. ${ }^{9-11}$ The prognosis is excellent if the disease is limited to skin and diagnosed promptly. The diagnosis of drug-induced LCV is sometimes challenging. However, the pattern of development and resolution of the skin lesions with discontinuation of culprit drugs can help in diagnosis. Drug-induced LCV should be suspected in any patient with small vessel vasculitis. For an exact diagnosis, history and examination, a temporal relationship with the offending drug(s), and exclusion of other potential causes are required. ${ }^{12}$ Biopsy of skin lesions for histopathology and examination under immunofluorescence are the gold standard. ${ }^{5}$ Perivascular inflammatory cell invasion with necrosis of the vessel wall and with deposition of fibrinoid material is pathognomonic for leukocytoclastic vasculitis (Figure-2). This was seen in our case. ${ }^{13}$

Management of LCV requires treatment of the underlying cause, discontinuation of suspected medications, and topical treatment of skin lesions with corticosteroids. Systemic agents, such as potent corticosteroids, colchicine, dapsone, nonsteroidal anti-inflammatory drugs, potassium iodide, antihistamines, immunosuppressive agents and rarely monoclonal antibodies are used, depending on the severity of the systemic vasculitis. ${ }^{10}$ Drug-induced LCV often resolves with discontinuation of the culprit on drug. ${ }^{14}$

Tigecycline is a tetracycline derivative commonly used to treat complicated skin and soft tissue infections. Reports of tetracycline derivative (e.g., minocycline) induced autoimmunity include druginduced lupus, cutaneous polyarteritis nodosa, and anti-nuclear cytoplasmic antibody (ANCA)-associated vasculitis. ${ }^{15}$ In our patient, tigecycline was the probable cause of LCV, and this is the first reported case to our knowledge. Forty-eight hours after the discontinuation of tigecycline, the patient's skin lesions started improving; after four days they resolved completely. Later the patient developed fatal pancreatitis (a well known side-effect of tigecycline) with multiorgan failure from which he did not survive.

In summary, drug-induced LCV should be suspected in any patient with a new onset skin rash, a temporal relationship with the offending drug, and resolution of symptoms with discontinuation of the drug. Physician should know that tigecycline-induced $\mathrm{LCV}$ is one of the rare side effects of this medication.

\footnotetext{
Author Affiliation: Kalpana Bhairavarasu is a faculty member in Rheumatology at Texas Tech University Health Sciences Center in Odessa, TX. Jaya Amaram, Ena Sharma, and Imran Umer are residents in Internal Medicine at TTUHSC in Odessa, TX. Satish Mocherla is a clinical assistant professor in Infectious Disease at TTUHSC in Odessa, TX. Philip A Conlin is a pathologist at DX Laboratories in Midland, TX.

Received: $10 / 23 / 2014$

Accepted: 01/02/2015

Reviewers: Anoop Nambiar MD

Published electronically: 01/15/2015

Conflict of Interest Disclosures: none
}

\section{REFERENCES}

1. Jennette JC, Falk RJ. Small-vessel vasculitis. $N$ Engl $J$ Med 1997; 337:1512-23.

2. Lotti T, Ghersetich I, Comacchi C, Jorizzo JL. Cutaneous small-vessel vasculitis. J Am Acad Dermatol 1998; 39:667-87. 
3. Hunder, GG, Arend WP, Bloch DA, Calabreso LH, Fauci AS, Fries JF, et al. Am Coll Rheum 1990 criteria for the classification of vasculitis. Arthritis Rheum 1990; 33:1135-6.

4. Ha YJ, Han YJ, Choi YW, Myung KB, Choi HY. Sibutramine induced cutaneous leukocytoclastic vasculitis: A case report. Ann Dermatol 2011; 23:544-7.

5. Lasić $\mathrm{D}$, Ivanišević $\mathrm{R}$, Uglešić $\mathrm{B}$, Cvitanović $M Z$, Glučina $\mathrm{D}$, Hlevnjak I. Valproate-acid induced cutaneous leukocytoclastic vasculitis. Psychiatr Danub 2012; 24(2):215-8.

6. Van Rosum AP, Pas HH, Fazzini F, Huitema MG, Limburg PC, Jonkman MF, et al. Abundance of the long pentraxin PTX3 at sites of leukocytoclastic lesions in patients with small-vessel vasculitis. Arthritis Rheum 2006; 54:986-91.

7. Mullick FG, McAllister HA Jr, Wagner BM, Fenoglio JJ Jr. Drug related vasculitis. Clinico- pathologic correlations in $30 \mathrm{pa}-$ tients. Hum Pathol 1979; 10:313-25

8. Jessop SJ. Cutaneous leukocytoclastic vasculitis: a clinical and etiological study. Brit J Rheum 1995; 34(10):942-5.

9. Roujeau JC, Stern RS. Severe adverse cutaneous reactions to drugs. N Engl J Med 1994; 331:1272-85.

10. Dubost JJ, Souteyrand P, Sauvezie B. Drug-induced vasculitides. Baillieres Clin Rheum 1991; 5:119-38

11. Jain KK. Drug-induced cutaneous vasculitis. Adverse Drug React Toxicol Rev 1993; 12:263-76

12. Wiik A. Drug-induced vasculitis. Curr Opin Rheum 2008; 20:35-9.

13. Carlson JA, Ng BT, Chen KR. Cutaneous vasculitis update: diagnostic criteria, classification, epidemiology, etiology, pathogenesis, evaluation and prognosis. Am J Dermatopathol 2005; 27:504-528.

14. Maunz G, Conzett T, Zimmerli W. Cutaneous vasculitis associated with fluoroquinolones. Infection 2009; 37(5):466-8.

15. Kermani TA, Ham EK, Camilleri MJ, Warrington KJ. Polyarteritis nodosa-like vasculitis in association with minocycline use: a single-center case series. Semin Arthiritis Rheum 2012; 42(2):213-21. 